\title{
Evaluation of some Novel Mode of Insecticides against Cotton Jassid (Amrasca biguttula biguttula Ishida) in New Alluvial Zone of West Bengal, India
}

\author{
Pronobesh Halder ${ }^{*}$, Madhab Kumar Datta ${ }^{2}$ and Sourav Basak ${ }^{1}$ \\ ${ }^{1}$ Department of Agricultural Entomology, Bidhan Chandra Krishi Viswavidyalaya, Mohanpur, \\ Nadia-741252, West Bengal, India \\ ${ }^{2}$ Department of Agronomy, Faculty of Agriculture, Bidhan Chandra Krishi Viswavidyalaya, \\ Mohanpur, Nadia-741252, West Bengal, India \\ *Corresponding author
}

\begin{tabular}{|l|}
\hline Ke y w or d s \\
Cotton, Jassid, \\
Insecticides \\
\hline Article Info \\
\hline Accepted: \\
04 March 2018 \\
Available Online: \\
10 April 2018 \\
\hline
\end{tabular}

\section{Introduction}

Cotton (Gossypium spp. L.) is an important fiber yielding crop of global importance, which is grown in tropical and subtropical regions of more than 80 countries the world over, contributes about $85 \%$ to the global Cotton production. In India, cotton is grown in about 118.72 lakh hectare with total production of 30.15 million tonnes (http://agricoop.nic.in/, 2017). Even though India ranks first with respect to area under cultivation, it stands third in total production,
Field experiments were conducted in two crop seasons during 2014 and 2015 at Bidhan Chandra Krishi Viswavidyalaya, Kalyani, West Bengal to evaluate the efficacy of some novel mode of insecticides like Buprofezin 25\% SC, Flonicamid 50\% WG, Spiromesifen $22.9 \%$ SC, Spirotetramat 240 SC, Sulfoxaflor 24\% SC, Clothianidin 50\% WDG, Dinotefuran 20\% SG along with some standard chemicals, Imidacloprid 17.8\% SL @ 0.5 $\mathrm{ml} / \mathrm{l}$ and Thiamethoxam 25\% WG @ $0.3 \mathrm{gm} / \mathrm{l}$ against Jassid [Amrasca biguttula biguttula (Ishida)] infesting cotton variety Surabhi. Nine numbers of insecticides with untreated control, altogether ten treatments replicated three times were done in the experiment. Results revealed that all the treated plots gave significant reduction of jassid population while there was a sharp increase in pest population in the untreated control. Among the treatments, the most effective insecticide was Buprofezin 25\% SC @ $1.6 \mathrm{ml} / 1$ (80.16\%) followed by Imidacloprid 17.8\% SL @ $0.5 \mathrm{ml} / 1$ (78.77\%) and least effective was Sulfoxaflor 24\% SC @ 0.3ml/1 (69.45\%). 
pests and chewing insect pests. Important sucking insect pests are whitefly, Bemisia tabaci (Genn.); thrips, Thrips tabaci (Lind.); jassid, Amrasca devastans (Dist.) and aphid, Aphis gossypii (Glov.) which are also designated as key pests causing most of the damage to the cotton crop. In Southeast Asian countries, jassid, Amrasca biguttula biguttula (Ishida), is one of the major pests of cotton. It is reported to cause $24.45 \%$ reduction in cotton yield (Bhat et al., 1986). Its incidence results in the loss of plant vigour, toxemia, hopper burn and spreads the mosaic virus diseases affecting fruit yield perceptibility (Samal and Patnaik, 2008; Shivanna et al., 2009). There are different pest control tactics, but the most common and quicker one is that of chemical control which is generally adopted by our farming community. Chemical control of the pests becomes imperative when all other control methods fail to control the target pests. Keeping in view the above information against sucking insect-pests the present investigation was conducted to evaluate the efficacy of some new group of insecticides against cotton jassid.

\section{Materials and Methods}

The field experiment was conducted at CBlock farm of Bidhan Chandra Krishi Viswavidyalaya, Kalyani, West Bengal, during rabi season of 2014 and 2015. The farm is located at $23.5^{\circ} \mathrm{N}$ latitude, $89^{\circ} \mathrm{E}$ longitude and $9.75 \mathrm{~m}$ above MSL. The soil of the experimental site was sandy loam with $\mathrm{pH}$ 6.8 and irrigation facility. Attempts were made to evaluate the effect of nine insecticides with their recommended doses viz. Buprofezin 25\% SC @ 1.6 ml/l, Flonicamid 50\% WG@0.4 gm/l, Thiamethoxam 25\% WG @ 0.3 gm/l, Spiromesifen 22.9\% SC @ $1 \mathrm{gm} / \mathrm{l}$, Spirotetramat $240 \quad \mathrm{SC}$ @ $0.75 \quad 0.7 \mathrm{ml} / \mathrm{l}$, Imidacloprid $17.8 \% \quad \mathrm{SL} \quad$ @ $\quad 0.5 \quad \mathrm{ml} / \mathrm{l}$, Sulfoxaflor 24\% SC @ 0.3 ml/l, Dinotefuran 20\% SG@0.3 gm/1 and Clothianidin 50\%
WDG @ 0.25 gm/1 with untreated control against cotton jassid (cultivar- Surabhi). The experiment was laid out in Randomized Block Design (RBD) with ten treatments including control and each treatment was replicated three times. The control plot was sprayed with water. The experimental plot size was $4 \mathrm{~m} x$ $3 \mathrm{~m}$ and the crop was grown on a row spacing of $75 \mathrm{~cm} \times 60 \mathrm{~cm}(\mathrm{R} \times \mathrm{P})$ under normal practices. All the sprayings were done using knapsack sprayer @ 5 litre of spray solution/ha at an interval of fifteen days. Three plants of each plot were randomly selected and tagged. The population of jassid was recorded from three leaf (upper, middle and lower) per plant before and 1, 3, 7 and 10 days after each spray.

\section{Results and Discussion}

In the year 2014 and 2015, the pre-treatment population of jassid varied from 2.11 - 3.89 and $2.22-5.56$ per leaf respectively. In both the year, the variation among the treatments was insignificant, but after each spray, significant reduction of jassid was observed. In first year (2014), highest mean per cent reduction of jassid after first spray was found in Buprofezin 25\% SC (85.08\%) followed by Dinotefuran 20\% SG @ 0.3 gm/1 (80.18\%) and Flonicamid 50\% WG $@ 0.4$ gm/l $(66.07 \%)$ was least effective. After second spray, highest mean per cent reduction was noted in Thiamethoxam 25\% WG @ 0.3 gm/1 $(87.96 \%)$ followed by Imidacloprid $17.8 \%$ SL @ $0.5 \mathrm{ml} / 1(82.40 \%)$ and lowest was in Sulfoxaflor 24\% SC @ $0.3 \mathrm{ml} / 1$ (68.50\%) treated plot. After third spray, highest mean per cent reduction was noted in Clothianidin $50 \%$ WDG @ $0.25 \mathrm{gm} / 1$ (84.05\%) followed by Imidacloprid $17.8 \% \quad \mathrm{SL} \quad @ \quad 0.5$ ml/l $(82.40 \%)$. The overall highest mean per cent reduction of jassid in the year 2014 was noted in Buprofezin 25\% SC @ $1.6 \mathrm{ml} / 1$ (80.42\%) followed by Imidacloprid17.8\% SL @ 0.5 ml/1 $(80.34 \%)$ treated plot as shown in Table 1. 


\section{Int.J.Curr.Microbiol.App.Sci (2018) 7(4): 33-37}

Table.1 Effect of some newer chemicals against jassid (Amrasca biguttula biguttula) on cotton (Pulled data of 2014)

\begin{tabular}{|c|c|c|c|c|c|c|c|c|c|c|c|c|c|c|c|c|c|c|c|}
\hline \multirow[t]{2}{*}{ Treatment } & \multirow{2}{*}{$\begin{array}{c}\text { Pre- } \\
\text { count } \\
\text { (No/ leaf) }\end{array}$} & \multicolumn{5}{|c|}{$\begin{array}{l}\text { Percentage reduction/increase }(+) \text { after first spray } \\
\text { over pre-treatment count }\end{array}$} & \multirow{2}{*}{$\begin{array}{c}\text { Pre } \\
\text { count } \\
\text { (N0/ } \\
\text { leaf) } \\
\text { Before } \\
2^{\text {nd }} \\
\text { spray }\end{array}$} & \multicolumn{5}{|c|}{$\begin{array}{l}\text { Percentage reduction/ increase }(+) \text { after second } \\
\text { spray over pre-treatment count }\end{array}$} & \multirow{2}{*}{$\begin{array}{c}\text { Pre } \\
\text { count } \\
\text { (No/ } \\
\text { leaf) } \\
\text { Before } \\
3^{\text {nd }} \\
\text { spray }\end{array}$} & \multicolumn{5}{|c|}{$\begin{array}{l}\text { Percentage reduction/ increase }(+) \text { after third spray } \\
\text { over pre-treatment count }\end{array}$} & \multirow{2}{*}{$\begin{array}{l}\text { Over all } \\
\text { mean \% } \\
\text { reductior }\end{array}$} \\
\hline & & $1 \mathrm{DS}^{*}$ & 3 DS* & 7 DS* & 10 DS* & Mean & & 1 DS* & 3 DS* & 7 DS* & 10 DS* & Mean & & 1 DS* & 3 DS* & 7 DS* & 10 DS* & Mean & \\
\hline $\begin{array}{l}\text { Buprofezin } \\
25 \% \text { SC }\end{array}$ & 2.22 & $\begin{array}{c}62.45 \\
(52.19)\end{array}$ & $\begin{array}{c}90.04 \\
(71.57)\end{array}$ & $\begin{array}{c}98.82 \\
(84.24)\end{array}$ & $\begin{array}{c}89.01 \\
(83.37)\end{array}$ & 85.08 & 1.65 & $\begin{array}{c}28.99 \\
(32.55)\end{array}$ & $\begin{array}{c}94.11 \\
(75.92)\end{array}$ & $\begin{array}{c}88.63 \\
(70.31)\end{array}$ & $\begin{array}{c}89.03 \\
(70.67)\end{array}$ & 75.02 & 2.02 & $\begin{array}{c}43.00 \\
(40.95)\end{array}$ & $\begin{array}{c}91.11 \\
(72.64)\end{array}$ & $\begin{array}{c}96.39 \\
(79.01)\end{array}$ & $\begin{array}{c}94.15 \\
(76.39)\end{array}$ & 81.16 & 80.42 \\
\hline $\begin{array}{l}\text { Flonicamid 50\% } \\
\text { WG }\end{array}$ & 2.55 & $\begin{array}{c}88.62 \\
(70.26)\end{array}$ & $\begin{array}{c}68.88 \\
(56.07)\end{array}$ & $\begin{array}{c}49.99 \\
(44.97)\end{array}$ & $\begin{array}{c}56.80 \\
(45.54)\end{array}$ & 66.07 & 5.74 & $\begin{array}{c}81.99 \\
(64.86)\end{array}$ & $\begin{array}{c}81.32 \\
(64.32)\end{array}$ & $\begin{array}{c}76.66 \\
(61.08)\end{array}$ & $\begin{array}{c}79.18 \\
(62.60)\end{array}$ & 79.78 & 6.42 & $\begin{array}{c}72.34 \\
(58.24)\end{array}$ & $\begin{array}{c}78.11 \\
(62.09)\end{array}$ & $\begin{array}{c}80.99 \\
(64.12)\end{array}$ & $\begin{array}{l}66.75 \\
(54.76)\end{array}$ & 74.54 & 73.46 \\
\hline $\begin{array}{l}\text { Thiamethoxam } \\
\text { 25\% WG }\end{array}$ & 2.67 & $\begin{array}{c}88.04 \\
(69.73)\end{array}$ & $\begin{array}{c}86.39 \\
(68.32)\end{array}$ & $\begin{array}{c}60.00 \\
(50.76)\end{array}$ & $\begin{array}{c}75.89 \\
(50.76)\end{array}$ & 75.58 & 5.22 & $\begin{array}{c}94.88 \\
(76.89)\end{array}$ & $\begin{array}{c}89.12 \\
(70.72)\end{array}$ & $\begin{array}{l}88.30 \\
(69.97)\end{array}$ & $\begin{array}{c}79.55 \\
(63.10)\end{array}$ & 87.96 & 4.09 & $\begin{array}{c}87.84 \\
(69.56)\end{array}$ & $\begin{array}{c}86.90 \\
(67.92)\end{array}$ & $\begin{array}{c}65.48 \\
(53.99)\end{array}$ & $\begin{array}{c}47.35 \\
(43.46)\end{array}$ & 71.89 & 78.47 \\
\hline $\begin{array}{c}\text { Spiromesifen } \\
22.9 \% \text { SC }\end{array}$ & 3.89 & $\begin{array}{c}78.27 \\
(62.20)\end{array}$ & $\begin{array}{c}70.90 \\
(57.38)\end{array}$ & $\begin{array}{c}77.24 \\
(61.48)\end{array}$ & $\begin{array}{c}75.11 \\
(61.49)\end{array}$ & 75.98 & 7.32 & $\begin{array}{c}62.63 \\
(52.29)\end{array}$ & $\begin{array}{l}83.00 \\
(65.64)\end{array}$ & $\begin{array}{l}80.44 \\
(63.73)\end{array}$ & $\begin{array}{c}82.42 \\
(65.18)\end{array}$ & 77.12 & 3.42 & $\begin{array}{c}76.87 \\
(61.23)\end{array}$ & $\begin{array}{c}69.18 \\
(56.27)\end{array}$ & $\begin{array}{c}75.15 \\
(60.15)\end{array}$ & $\begin{array}{c}76.01 \\
(60.64)\end{array}$ & 74.30 & 75.51 \\
\hline $\begin{array}{c}\text { Imidacloprid } \\
17.8 \% \mathrm{SL}\end{array}$ & 3.22 & $\begin{array}{c}85.03 \\
(67.40)\end{array}$ & $\begin{array}{c}60.00 \\
(50.76)\end{array}$ & $\begin{array}{c}84.41 \\
(66.83)\end{array}$ & $\begin{array}{c}86.03 \\
(66.71)\end{array}$ & 78.86 & 5.92 & $\begin{array}{c}80.22 \\
(63.56)\end{array}$ & $\begin{array}{c}82.14 \\
(64.99)\end{array}$ & $\begin{array}{c}85.61 \\
(67.68)\end{array}$ & $\begin{array}{c}81.64 \\
(64.40)\end{array}$ & 82.40 & 5.63 & $\begin{array}{c}84.00 \\
(66.50)\end{array}$ & $\begin{array}{r}82.69 \\
(65.38)\end{array}$ & $\begin{array}{c}70.90 \\
(58.60)\end{array}$ & $\begin{array}{c}81.62 \\
(64.59)\end{array}$ & 79.78 & 80.34 \\
\hline $\begin{array}{l}\text { Sulfoxaflor } 24 \% \\
\text { SC }\end{array}$ & 3.11 & $\begin{array}{c}65.07 \\
(53.78)\end{array}$ & $\begin{array}{c}75.52 \\
(60.32)\end{array}$ & $\begin{array}{c}72.06 \\
(58.06)\end{array}$ & $\begin{array}{c}78.72 \\
(58.07)\end{array}$ & 72.84 & 5.81 & $\begin{array}{c}60.06 \\
(50.79)\end{array}$ & $\begin{array}{c}72.98 \\
(58.66)\end{array}$ & $\begin{array}{c}70.99 \\
(53.38)\end{array}$ & $\begin{array}{c}70.00 \\
(56.77)\end{array}$ & 68.50 & 6.04 & $\begin{array}{c}62.22 \\
(52.05)\end{array}$ & $\begin{array}{c}68.09 \\
(55.58)\end{array}$ & $\begin{array}{c}73.23 \\
(58.84)\end{array}$ & $\begin{array}{c}75.29 \\
(60.24)\end{array}$ & 69.70 & 70.34 \\
\hline $\begin{array}{l}\text { Dinotefuran } \\
20 \% \text { SG }\end{array}$ & 2.11 & $\begin{array}{c}70.92 \\
(57.34)\end{array}$ & $\begin{array}{c}78.85 \\
(62.61)\end{array}$ & $\begin{array}{c}84.42 \\
(66.72)\end{array}$ & $\begin{array}{c}86.55 \\
(66.83)\end{array}$ & 80.18 & 3.91 & $\begin{array}{c}80.11 \\
(63.49)\end{array}$ & $\begin{array}{c}78.00 \\
(62.00)\end{array}$ & $\begin{array}{c}86.00 \\
(68.00)\end{array}$ & $\begin{array}{c}75.29 \\
(60.16)\end{array}$ & 79.85 & 4.32 & $\begin{array}{c}77.62 \\
(61.74)\end{array}$ & $\begin{array}{c}79.00 \\
(62.70)\end{array}$ & $\begin{array}{c}81.01 \\
(64.14)\end{array}$ & $\begin{array}{l}81.92 \\
(64.81)\end{array}$ & 79.88 & 79.97 \\
\hline $\begin{array}{l}\text { Clothianidin } \\
\text { 50\% WDG }\end{array}$ & 2.78 & $\begin{array}{c}70.00 \\
(56.76)\end{array}$ & $\begin{array}{c}75.62 \\
(60.38)\end{array}$ & $\begin{array}{c}73.59 \\
(59.05)\end{array}$ & $\begin{array}{c}72.64 \\
(59.05)\end{array}$ & 72.96 & 4.67 & $\begin{array}{c}79.06 \\
(62.75)\end{array}$ & $\begin{array}{c}83.59 \\
(66.07)\end{array}$ & $\begin{array}{c}75.43 \\
(60.26)\end{array}$ & $\begin{array}{c}78.00 \\
(62.01)\end{array}$ & 79.02 & 5.62 & $\begin{array}{c}79.54 \\
(63.08)\end{array}$ & $\begin{array}{c}83.00 \\
(65.67)\end{array}$ & $\begin{array}{c}86.67 \\
(68.55)\end{array}$ & $\begin{array}{c}86.99 \\
(68.86)\end{array}$ & 84.05 & 78.67 \\
\hline $\begin{array}{l}\text { Untreated } \\
\text { control }\end{array}$ & 3.33 & +09.48 & +34.92 & +40.81 & +37.44 & +30.66 & 7.58 & +52.55 & +67.00 & +58.03 & +38.39 & +53.99 & 8.01 & +11.99 & +43.45 & +33.39 & +63.42 & +38.06 & \\
\hline $\begin{array}{c}\text { S.Em }( \pm) \\
\text { C.D. at } 5 \%\end{array}$ & & $\begin{array}{l}1.06 \\
3.17\end{array}$ & $\begin{array}{l}0.94 \\
2.81\end{array}$ & $\begin{array}{l}1.06 \\
3.18\end{array}$ & $\begin{array}{l}0.89 \\
2.68\end{array}$ & & & $\begin{array}{l}0.68 \\
2.03\end{array}$ & $\begin{array}{l}0.45 \\
1.36\end{array}$ & $\begin{array}{l}0.43 \\
1.29\end{array}$ & $\begin{array}{l}0.48 \\
1.44\end{array}$ & & & $\begin{array}{l}0.71 \\
2.14\end{array}$ & $\begin{array}{l}0.52 \\
1.56\end{array}$ & $\begin{array}{l}0.75 \\
2.25\end{array}$ & $\begin{array}{l}0.69 \\
2.09\end{array}$ & & \\
\hline
\end{tabular}




\section{Int.J.Curr.Microbiol.App.Sci (2018) 7(4): 33-37}

Table.2 Effect of some newer chemicals against jassid (Amrasca biguttula biguttula) on cotton (Pulled data of 2015)

\begin{tabular}{|c|c|c|c|c|c|c|c|c|c|c|c|c|c|c|c|c|c|c|c|c|}
\hline \multirow[t]{2}{*}{ Treatment } & \multirow{2}{*}{$\begin{array}{l}\text { Pre- } \\
\text { count } \\
\text { (No/ } \\
\text { leaf) }\end{array}$} & \multicolumn{5}{|c|}{$\begin{array}{l}\text { Percentage reduction/increase ( }+ \text { ) after first spray } \\
\text { over pre-treatment count }\end{array}$} & \multirow{2}{*}{$\begin{array}{c}\text { Pre } \\
\text { count } \\
\text { (N0/ } \\
\text { leaf) } \\
\text { Before } \\
2^{\text {nd }} \\
\text { spray }\end{array}$} & \multicolumn{5}{|c|}{$\begin{array}{l}\text { Percentage reduction/ increase }(+) \text { after second } \\
\text { spray over pre-treatment count }\end{array}$} & \multirow{2}{*}{$\begin{array}{c}\text { Pre } \\
\text { count } \\
\text { (N0/ } \\
\text { leaf) } \\
\text { Before } \\
\mathbf{3}^{\text {nd }} \\
\text { spray }\end{array}$} & \multicolumn{5}{|c|}{$\begin{array}{l}\text { Percentage reduction/ increase ( }+ \text { ) after third spray } \\
\text { over pre-treatment count }\end{array}$} & \multirow[t]{2}{*}{$\begin{array}{l}\text { Mean } \% \\
\text { reduction }\end{array}$} & \multirow{2}{*}{$\begin{array}{l}\text { Over all } \\
\text { mean \% } \\
\text { reduction }\end{array}$} \\
\hline & & $1 \mathrm{DS} *$ & 3 DS* & 7 DS* & 10 DS* & Mean & & $1 \mathrm{DS} *$ & 3 DS* & 7 DS* & 10 DS* & Mean & & 1 DS* & 3 DS* & 7 DS* & $10 \mathrm{DS}^{*}$ & Mean & & \\
\hline $\begin{array}{l}\text { Buprofezin } 25 \% \\
\text { SC }\end{array}$ & 4.00 & $\begin{array}{c}55.64 \\
(48.23)\end{array}$ & $\begin{array}{c}92.86 \\
(74.47)\end{array}$ & $\begin{array}{c}89.72 \\
(71.28)\end{array}$ & $\begin{array}{c}96.07 \\
(78.53)\end{array}$ & 83.57 & 2.24 & $\begin{array}{c}37.62 \\
(37.81)\end{array}$ & $\begin{array}{c}92.23 \\
(73.78)\end{array}$ & $\begin{array}{c}96.00 \\
(78.49)\end{array}$ & $\begin{array}{c}88.95 \\
(70.55)\end{array}$ & 78.70 & 2.02 & $\begin{array}{c}27.92 \\
(31.86)\end{array}$ & $\begin{array}{c}90.47 \\
(71.99)\end{array}$ & $\begin{array}{c}96.55 \\
(79.26)\end{array}$ & $\begin{array}{c}95.01 \\
(77.23)\end{array}$ & 77.48 & 79.91 & 80.16 \\
\hline $\begin{array}{l}\text { Flonicamid 50\% } \\
\text { WG }\end{array}$ & 4.00 & $\begin{array}{c}82.78 \\
(65.48)\end{array}$ & $\begin{array}{c}79.86 \\
(63.30)\end{array}$ & $\begin{array}{c}55.80 \\
(48.31)\end{array}$ & $\begin{array}{c}48.22 \\
(43.96)\end{array}$ & 66.66 & 2.57 & $\begin{array}{c}81.00 \\
(64.13)\end{array}$ & $\begin{array}{c}84.54 \\
(66.93)\end{array}$ & $\begin{array}{c}72.19 \\
(58.15)\end{array}$ & $\begin{array}{c}69.88 \\
(56.69)\end{array}$ & 76.90 & 3.42 & $\begin{array}{c}73.11 \\
(58.74)\end{array}$ & $\begin{array}{c}79.41 \\
(62.99)\end{array}$ & $\begin{array}{c}73.05 \\
(58.72)\end{array}$ & $\begin{array}{c}65.66 \\
(54.14)\end{array}$ & 72.80 & 72.12 & 72.79 \\
\hline $\begin{array}{l}\text { Thiamethoxam } \\
\text { 25\% WG }\end{array}$ & 5.44 & $\begin{array}{c}72.98 \\
(58.65)\end{array}$ & $\begin{array}{c}75.66 \\
(60.49)\end{array}$ & $\begin{array}{c}73.95 \\
(59.31)\end{array}$ & $\begin{array}{c}70.00 \\
(56.76)\end{array}$ & 73.14 & 3.21 & $\begin{array}{l}82.95 \\
(65.58)\end{array}$ & $\begin{array}{l}80.03 \\
(63.43)\end{array}$ & $\begin{array}{c}75.51 \\
(60.39)\end{array}$ & $\begin{array}{l}78.00 \\
(62.01)\end{array}$ & 79.12 & 3.09 & $\begin{array}{c}84.42 \\
(66.83)\end{array}$ & $\begin{array}{l}77.42 \\
(61.60)\end{array}$ & $\begin{array}{c}79.88 \\
(63.32)\end{array}$ & $\begin{array}{c}71.49 \\
(57.70)\end{array}$ & 78.30 & 76.85 & 77.66 \\
\hline $\begin{array}{l}\text { Spiromesifen } \\
22.9 \% \text { SC }\end{array}$ & 5.56 & $\begin{array}{c}66.81 \\
(64.80)\end{array}$ & $\begin{array}{c}71.71 \\
(57.84)\end{array}$ & $\begin{array}{c}78.93 \\
(62.65)\end{array}$ & $\begin{array}{c}78.00 \\
(62.01)\end{array}$ & 73.86 & 3.98 & $\begin{array}{c}56.00 \\
(48.42)\end{array}$ & $\begin{array}{c}73.43 \\
(58.97)\end{array}$ & $\begin{array}{c}79.99 \\
(63.40)\end{array}$ & $\begin{array}{c}83.13 \\
(65.72)\end{array}$ & 73.13 & 6.90 & $\begin{array}{c}67.37 \\
(55.15)\end{array}$ & $\begin{array}{c}78.82 \\
(62.57)\end{array}$ & $\begin{array}{c}80.16 \\
(63.64)\end{array}$ & $\begin{array}{c}81.26 \\
(64.32)\end{array}$ & 76.90 & 74.63 & 75.07 \\
\hline $\begin{array}{l}\text { Spirotetramat } \\
240 \text { SC }\end{array}$ & 3.45 & $\begin{array}{r}72.09 \\
(58.09)\end{array}$ & $\begin{array}{c}62.98 \\
(52.50)\end{array}$ & $\begin{array}{l}77.62 \\
(61.74)\end{array}$ & $\begin{array}{c}73.44 \\
(58.95)\end{array}$ & 71.53 & 2.78 & $\begin{array}{c}70.00 \\
(56.81)\end{array}$ & $\begin{array}{c}70.42 \\
(57.08)\end{array}$ & $\begin{array}{c}70.00 \\
(56.81)\end{array}$ & $\begin{array}{c}66.09 \\
(54.36)\end{array}$ & 69.12 & 5.55 & $\begin{array}{c}68.00 \\
(55.53)\end{array}$ & $\begin{array}{c}60.00 \\
(50.76)\end{array}$ & $\begin{array}{c}78.81 \\
(62.56)\end{array}$ & $\begin{array}{l}75.55 \\
(60.41)\end{array}$ & 70.59 & 70.41 & 72.05 \\
\hline $\begin{array}{l}\text { Imidacloprid } \\
17.8 \% \text { SL }\end{array}$ & 3.78 & $\begin{array}{c}80.32 \\
(63.63)\end{array}$ & $\begin{array}{c}70.66 \\
(57.18)\end{array}$ & $\begin{array}{c}78.92 \\
(62.64)\end{array}$ & $\begin{array}{c}75.02 \\
(60.06)\end{array}$ & 76.23 & 2.11 & $\begin{array}{c}82.98 \\
(65.60)\end{array}$ & $\begin{array}{c}78.77 \\
(62.53)\end{array}$ & $\begin{array}{c}72.00 \\
(58.03)\end{array}$ & $\begin{array}{c}68.05 \\
(55.55)\end{array}$ & 75.45 & 6.00 & $\begin{array}{c}84.87 \\
(67.20)\end{array}$ & $\begin{array}{c}82.22 \\
(65.05)\end{array}$ & $\begin{array}{c}79.66 \\
(63.18)\end{array}$ & $\begin{array}{c}73.13 \\
(58.77)\end{array}$ & 79.95 & 77.21 & 78.77 \\
\hline $\begin{array}{l}\text { Sulfoxaflor } 24 \% \\
\text { SC }\end{array}$ & 3.11 & $\begin{array}{c}70.62 \\
(57.15)\end{array}$ & $\begin{array}{c}64.99 \\
(53.70)\end{array}$ & $\begin{array}{l}78.14 \\
(62.10)\end{array}$ & $\begin{array}{c}69.39 \\
(56.38)\end{array}$ & 70.78 & 2.03 & $\begin{array}{c}65.00 \\
(53.74)\end{array}$ & $\begin{array}{l}77.69 \\
(61.78)\end{array}$ & $\begin{array}{c}70.90 \\
(57.36)\end{array}$ & $\begin{array}{c}60.91 \\
(51.28)\end{array}$ & 68.62 & 4.09 & $\begin{array}{c}61.21 \\
(51.45)\end{array}$ & $\begin{array}{c}63.42 \\
(52.76)\end{array}$ & $\begin{array}{c}71.98 \\
(58.02)\end{array}$ & $\begin{array}{c}68.72 \\
(55.97)\end{array}$ & 66.33 & 68.57 & 69.45 \\
\hline $\begin{array}{l}\text { Dinotefuran } \\
20 \% \text { SG }\end{array}$ & 2.22 & $\begin{array}{c}78.77 \\
(62.53)\end{array}$ & $\begin{array}{c}70.80 \\
(57.32)\end{array}$ & $\begin{array}{c}80.01 \\
(63.41)\end{array}$ & $\begin{array}{c}72.09 \\
(58.08)\end{array}$ & 75.41 & 2.05 & $\begin{array}{c}72.63 \\
(58.43)\end{array}$ & $\begin{array}{c}81.30 \\
(64.36)\end{array}$ & $\begin{array}{c}70.55 \\
(57.13)\end{array}$ & $\begin{array}{c}83.72 \\
(66.17)\end{array}$ & 77.05 & 5.78 & $\begin{array}{c}75.62 \\
(60.46)\end{array}$ & $\begin{array}{c}81.00 \\
(64.13)\end{array}$ & $\begin{array}{c}83.24 \\
(65.86)\end{array}$ & $\begin{array}{c}78.09 \\
(62.06)\end{array}$ & 79.48 & 77.31 & 78.64 \\
\hline $\begin{array}{l}\text { Clothianidin } \\
\text { 50\% WDG }\end{array}$ & 2.78 & $\begin{array}{c}68.95 \\
(56.11)\end{array}$ & $\begin{array}{c}72.99 \\
(58.62)\end{array}$ & $\begin{array}{c}77.00 \\
(61.33)\end{array}$ & $\begin{array}{c}70.88 \\
(57.31)\end{array}$ & 72.88 & 1.96 & $\begin{array}{c}79.60 \\
(63.12)\end{array}$ & $\begin{array}{c}85.01 \\
(67.38)\end{array}$ & $\begin{array}{c}76.00 \\
(60.69)\end{array}$ & $\begin{array}{c}69.01 \\
(56.15)\end{array}$ & 77.40 & 8.40 & $\begin{array}{c}78.00 \\
(62.01)\end{array}$ & $\begin{array}{c}80.67 \\
(63.89)\end{array}$ & $\begin{array}{c}73.48 \\
(59.00)\end{array}$ & $\begin{array}{c}71.21 \\
(57.53)\end{array}$ & 75.84 & 75.37 & 77.02 \\
\hline $\begin{array}{l}\text { Untreated } \\
\text { control }\end{array}$ & 4.56 & +13.98 & +45.38 & +56.50 & +38.34 & +38.55 & 3.78 & +29.74 & +56.09 & +72.19 & +69.62 & +56.91 & 8.98 & +18.24 & +57.04 & +68.92 & +73.04 & +54.31 & & \\
\hline $\begin{array}{l}\text { S.Em }( \pm) \\
\text { C.D. at } 5 \%\end{array}$ & & $\begin{array}{l}0.73 \\
2.21\end{array}$ & $\begin{array}{l}0.96 \\
2.89\end{array}$ & $\begin{array}{l}0.48 \\
1.45\end{array}$ & $\begin{array}{l}0.75 \\
2.25\end{array}$ & & & $\begin{array}{l}0.89 \\
2.67\end{array}$ & $\begin{array}{l}1.07 \\
3.21\end{array}$ & $\begin{array}{l}1.23 \\
3.68\end{array}$ & $\begin{array}{l}0.30 \\
0.91\end{array}$ & & & $\begin{array}{l}1.20 \\
3.59\end{array}$ & $\begin{array}{l}0.72 \\
2.16\end{array}$ & $\begin{array}{l}1.06 \\
3.18\end{array}$ & $\begin{array}{l}1.13 \\
3.38\end{array}$ & & & \\
\hline
\end{tabular}


In second year (2015), same results were obtained after first and second spray i.e., highest mean per cent reduction of jassid was buprofezin $25 \%$ SC $(83.57 \%)$ and Thiamethoxam 25\%WG @0.3 gm/1 (79.12\%), respectively. After third spray, highest mean per cent reduction was noted in Imidacloprid $17.8 \%$ SL@ $0.5 \mathrm{ml} / 1$ (79.97\%). The overall highest mean per cent reduction of jassid in the year 2015 was noted in Buprofezin 25\% SC @ 1.6 $\mathrm{ml} / 1(79.91 \%)$ followed by Sulfoxaflor $24 \% \mathrm{SC}$ @ $0.3 \mathrm{ml} / \mathrm{l}(77.31 \%)$ treated plot as shown in Table 2.

So, the average overall highest mean per cent reduction of jassid in both the year was noted in Buprofezin 25\% SC @ $1.6 \mathrm{ml} / \mathrm{l}(80.16 \%)$ followed by Imidacloprid 17.8\% SL @ 0.5 ml/1 $(78.77 \%)$ treated plot as shown in Table 2. In an earlier study, Mandal et al., (2014) found that the order of bio-efficacy on the basis of per cent reduction of cotton jassid over control plot was: buprofezin > imidacloprid > spiromesifen. Abbas et al., 2012 reported that imidacloprid proved to be highly effective against Jassid and whitefly.

We can conclude that the test chemical Buprofezin 25\% SC @ 1.6 ml/l may be incorporated in the integrated management practices of cotton jassid. Sulfoxaflor $24 \%$ SC @ $0.3 \mathrm{ml} / \mathrm{1}$, though an effective systemic insecticide against different sucking pests of crops, has been proved as the least effective in terms of reduction of jassid infestation in cotton.

\section{Acknowledgments}

The authors are grateful to Professor and Head, Department of Agricultural Entomology, Bidhan Chandra Krishi Viswavidyalaya,
Mohanpur, Nadia for providing necessary facilities to carry out the present investigations.

\section{References}

Abbas, Q., Arif, M. J., Gogi, M. D., Abbas, S. K. and Karar, H. 2012. Performance of Imidacloprid, Thiomethoxam, Acetamaprid and a Biocontrol Agent Chrysoperla carnea) Against Whitefly, Jassid and Thrips on Different Cotton Cultivars. World Journal of Zoology. 7(2): 141-146.

Anonymous. 2004. Annual Report. All India coordinated cotton improvement project, Coimbatore. 1-12.

Bhat, A., Soomro, A. and Mallah, G. H. 1986. Evaluation of some cotton varieties with known genetic marker for their resistant against sucking insect pest boll worm complex. Turkiye Butki Kurume Dergisi. 6(2): 3-14.

http://agricoop.nic.in/, 2017.

Mandal, D., Bhowmik, P. and Chatterjee, M. L. 2014. Effect of Newer Insecticides against White Fly, Bemisia tabaci (Gennadius) and Jassid, Amrasca biguttula biguttula (Ishida) on Cotton. Pesticide Research Journal. 25(2): 117-122.

Naqvi, K. M. 1976. Crop protection to boost up cotton production. Proc. Cotton Production Seminar, Organized by ESSO Fert. Co. Ltd., Pak. pp. 119-125.

Samal, T. and Patnaik, H.P 2008. Field efficacy of insecticides against Amrasca bigttula biguttula (Ishida) incidence on eggplant. Annals of Plant Protection Science, 16(1): 115- 118

Shivanna, B. K., Nagraja, D. N., Manjunatha, M., Gayathridevi, S., Pradeep, S. and Grijesh, G. K. 2009. Bionomics of leafhopper Amrasca bigutulla biguttula (Ishida) on transgenic Bt. Cotton. Karnataka Journal of Agricultural Science. 22(3Spl. Issue): 538540 .

\section{How to cite this article:}

Pronobesh Halder, Madhab Kumar Datta and Sourav Basak. 2018. Evaluation of some Novel Mode of Insecticides against Cotton Jassid (Amrasca biguttula biguttula Ishida) in New Alluvial Zone of West Bengal, India. Int.J.Curr.Microbiol.App.Sci. 7(04): 33-37. doi: https://doi.org/10.20546/ijcmas.2018.704.004 